\title{
TRACE-LIKE FUNCTIONS ON RINGS WITH NO NILPOTENT ELEMENTS
}

BY

\author{
M. COHEN AND SUSAN MONTGOMERY ${ }^{1}$
}

\begin{abstract}
Let $R$ be a ring with no nilpotent elements, with extended center $C$, and let $E$ be the set of idempotents in $C$. Our first main result is that for any finite group $G$ acting as automorphisms of $R$, there exist a finite set $L \subseteq E$ and an $R^{G}$-bimodule homomorphism $\tau: R \rightarrow(R L)^{G}$ such that $\tau(R)$ is an essential ideal of $(R E)^{G}$. This theorem is applied to show the following: if $R$ is a Noetherian, affine $P I$-algebra (with no nilpotent elements) over the commutative Noetherian ring $A$, and $G$ is a finite group of $A$-automorphisms of $R$ such that $R^{G}$ is Noetherian, then $R^{G}$ is affine over $A$.
\end{abstract}

0. Introduction and definitions. Let $R$ be a semiprime ring, $G$ a finite group of $\operatorname{order}|G|$ acting as automorphisms on $R$, and $R^{G}=\left\{r \in R \mid r^{g}=r\right.$, all $\left.g \in G\right\}$, the fixed ring of $G$ on $R$. The trace of $G$ on $R$ is the function $t_{G}: R \rightarrow R^{G}$ given by $t_{G}(x)=\sum_{g \in G} x^{g}$. It is clearly an $R^{G}$-bimodule homomorphism, and it is known that if either $R$ has no aditive $|G|$-torsion [3] or $G$ is " $X$-outer" on $R[15]$, then $0 \neq t_{G}(R)$ is an essential ideal of $R^{G}$. That is, $t_{G}$ behaves almost like a projection. The existence of such a nontrivial trace function has had a number of applications in studying the relationship between $R$ and $R^{G}$ (see, for example, $[5,8,15,17]$ ).

Is is this rather nice situation which we would like to imitate for rings with no nilpotent elements. The trace function itself will not work, since it is possible that $t_{G}(R) \equiv 0$, even when $R$ is a division ring [16]. When $R$ is a domain, an appropriate substitute exists for $t_{G}$, namely, a "partial trace function". For a subset $\Lambda \subseteq G$, let $t_{\Lambda}(x)=\sum_{g \in \Lambda} x^{g}$. If $t_{\Lambda}(R) \subseteq R^{G}, t_{\Lambda}$ is called a partial trace function; if also $t_{\Lambda}(R) \neq 0$, $t_{\Lambda}$ is nontrivial. It was proved in [16] that for any finite group $G$ acting on a domain $R$, there exists a nontrivial partial trace function $t_{\Lambda}$ for $G$ on $R$. This had been proved earlier for division rings in [8]. Clearly $t_{\Lambda}$ is an $R^{G}$-bimodule homomorphism, and $t_{\Lambda}(R)$ is essential in $R^{G}$ as $R^{G}$ is also a domain. Unfortunately, partial trace functions will not work for any ring with no nilpotent elements. If $G$ is solvable, or acts as inner automorphisms on $R$, then nontrivial partial trace functions exist [7]; however, for any nonsolvable group $G$, a ring $R$ with no nilpotent elements can be constructed such that all partial trace functions for $G$ on $R$ are trivial [9]. In

Received by the editors June 22, 1981.

1980 Mathematics Subject Classification. Primary 16A72, 16A74, 16A33; Secondary 13B05, 13B15, $16 \mathrm{~A} 38$.

${ }^{1}$ Both authors wish to thank the U.S.-Israel Binational Science Foundation for travel support; research of the second author was partially supported by NSF Grant No. MCS 76-07240. The second author would also like to thank the University of Leeds for its hospitality while part of this work was being done. 
addition, even when a nontrivial partial trace function exists, $t_{\Lambda}(R)$ may be only a small part of $R^{G}$, and not essential. Another trace-like function was constructed in [12]; it also has the disadvantage that its image can be very small, and in addition, the image of $R$ is in $Q_{0}(R)^{G}$, where $Q_{0}(R)$ is the Martindale quotient ring of $R$, and not in $R^{G}$ itself.

In this paper, we show that trace-like functions exist which have essential images, in a ring very close to $R^{G}$. Our main result is the following:

THEOREM 2.8. Let $R$ be a ring with no nilpotent elements, let $C$ be its extended center, and let $E$ be the set of idempotents in $C$. Let $G$ be any finite group acting as automorphisms on $R$. Then there exist a finite set $L \subseteq E$ and an $R^{G}$-bimodule homomorphism $\tau: R \rightarrow(R L)^{G}$ sch that $\tau(R)$ is essential in $(R E)^{G}$.

Moreover, there exists an essential ideal $K$ of $R$ such that $\tau(K) \subseteq R^{G}$ and is essential in $R^{G}$.

The construction of $\tau$ in this theorem specializes, in the case of a domain, to the construction of the partial trace function in [16].

In $\$ 3$ of the paper, we give an application of Theorem 2.8 to determining when certain fixed rings are affine (a ring $R$ is affine over a central subring $A$ if $R$ is finitely-generated as an $A$-algebra); this extends E. Noether's theorem on finite groups acting on commutative rings. Some results on this problem had already been obtained by L. W. Small and the second author [17]. Their first result is that if $R$ is Noetherian and affine over a commutative Noetherian ring $A$, and $|G|^{-1} \in R$, then $R^{G}$ is affine. Note that in this situation, the mapping $\rho(x)=|G|^{-1} t_{G}(x)$ is a projection of $R$ onto $R^{G}$. Their second result, with no assumptions on $|G|$, is the following: if $R$ is a Noetherian domain satisfying a polynomial identity $(P I)$, which is affine over a commutative Noetherian ring $A$, and $G$ is a finite group of $A$-automorphisms of $R$ such that $R^{G}$ is Noetherian, then $R^{G}$ is affine over $A$. The proof of this theorem used the partial trace function $t$ described above. It is not surprising that the proofs of these theorems use trace functions; classically, the existence of a projection from $R$ to $R^{G}$ played an important role in Hilbert's theorem that for $G=S L(m)$ acting on $R=k\left[x_{1}, \ldots, x_{n}\right], k$ of characteristic $0, R^{G}$ is affine over $k$ [18].

The second main theorem in the present paper extends the second result of Montgomery and Small to the case of semiprime PI rings.

THEOREM 3.3. Let $R$ be a semiprime Noetherian PI ring which is an affine algebra over a commutative Noetherian ring $A$. Let $G$ be a finite group of A-automorphisms of $R$ such that $R^{G}$ is Noetherian. Then $R^{G}$ is also affine over $A$, in any of the following cases:

(1) $R$ has no $|G|$-torsion,

(2) $G$ is $X$-outer on $R$,

(3) $R$ has no nilpotent elements. 
We note that the analog of Noether's theorem is not true for noncommutative rings in general; an example is given in [17] of a prime, Noetherian, affine, $P I$-algebra $R$ and a group $G$ of order $p$, a prime, such that $R^{G}$ is not affine. Another example is given there to show that even in characteristic 0 , the hypothesis that $R$ is Noetherian cannot be omitted. However, the hypothesis that $R^{G}$ is Noetherian may be superfluous (see comments at the end of $\$ 3$ below).

We now review the definitions used in this paper. For any semiprime ring $R$, let $\mathscr{F}=\mathscr{F}(R)$ denote the filter of essential two-sided ideals of $R$, and let $Q_{0}(R)$ $=\lim _{I \in \mathscr{F}} \operatorname{Hom}_{R}\left({ }_{R} I,{ }_{R} R\right)$, the Martindale quotient ring of $R \cdot Q_{0}(R)$ is also semiprime, and is prime when $R$ is prime. We may consider $R \subseteq Q_{0}(R)$ via $r \rightarrow f_{r}$, right multiplication by $r$. By construction, for any $0 \neq x \in R$, there exists $I \in \mathscr{F}(R)$ such that $0 \neq I x \subseteq R$. The extended center $C=C(R)$ of $R$ is the center of $Q_{0}(R)$ (it is also the center of the maximal ring of quotients). $Q_{0}(R)$ was defined for prime rings in [14] and for more general rings in [1].

Let $E=E(R)$ be the set of idempotents in $C$, and let $N=N(R)=\left\{x \in Q_{0}(R) \mid\right.$ $x R=R x\}$, the set of $R$-normalizing elements of $Q_{0}(R)$. The normal closure $R N$ of $R$ is the subring of $Q_{0}(R)$ generated by $R$ and $N(R)$; it was defined in [16] and studied further in [6]. When $R$ has no nilpotent elements, $R N$ also has no nilpotent elements [16]. The set $N$ has the structure of an inverse semigroup; that is, for each $x \in N$ there is a unique "inverse" $x^{\prime} \in N$ and $e=e_{x} \in E$ such that $x x^{\prime}=e=x^{\prime} x, e x=x$, and $e x^{\prime}=x^{\prime}[6]$.

For any $g \in \operatorname{Aut}(R), g$ has a unique extension to $Q_{0}(R)$. Let $\phi_{g}=\left\{x \in Q_{0}(R) \mid\right.$ $r x=x r^{g}$, all $\left.r \in R\right\}$; we say that $g$ is $X$-inner if $\phi_{g} \neq 0$. For any subgroup $G \subseteq \operatorname{Aut}(R), G_{\text {inn }}$ is the set of $X$-inner automorphisms in $G$. If $G_{\text {inn }}=\langle 1\rangle$, we say that $G$ is $X$-outer. It should be noted that $G_{\text {inn }}$ is not necessarily a subgroup of $G$, though it is closed under conjugation and under inverses. These definitions, generalizing the usual notion of inner automorphism, are due to Kharchenko [11], who also proves in [12] that for any $g \in \operatorname{Aut}(R)$ there exists $x_{g} \in \phi_{g}$ such that $\phi_{g}=C x_{g}$, a cyclic $C$-module. Now, clearly $\phi_{g} \subseteq N$, so we may work in $R N$ rather than $Q_{0}(R)$. In particular, $x_{g}$ has an inverse $x_{g}^{\prime}$ with $x_{g} x_{g}^{\prime}=e_{g} \in E$ by the above. We say that $e_{g}$ is the idempotent belonging to $g$. For any $h \in G$, it is straightforward to check that $\left(e_{g}\right)^{h}=e_{h^{-1} g h}$.

In the special case when $R$ is a semiprime Goldie ring with classical quotient ring $Q(R)$, the extended center $C(R)$ is just the center of $Q(R)$, and $g \in \operatorname{Aut}(R)$ is $X$-inner if and only if, when extended to $Q(R), g$ is genuinely inner on some nonzero ideal of $Q(R)$.

1. $X$-inner automorphisms of semiprime rings. Let $R$ be a semiprime ring. In this section we prove a number of technical lemmas which will be required in $\S 2$. These results concern certain subgroups of $G_{\text {inn }}$ which arise from central idempotents, so may be of independent interest. We will not require that $R$ have no nilpotent elements until the end of this section.

For an idempotent $e \in E(R)$, we summarize some properties of $e$ with respect to $Q_{0}, N, C$, and $E$, as defined in the introduction. Let $R e=\{r e \mid r \in R\} ; R e$ is a subring of $R E$. As the arguments are standard, we only outline the proofs. 
LEMMA 1.1. Let $e \in E(R)$. Then

(1) $Q_{0}(R e)=Q_{0}(R) e$,

(2) $N(R e) \supseteq N(R) e$,

(3) $C(R e)=C(R) e$,

(4) $E(R e)=E(R) e$,

(5) if $A$ is an $R-R$ submodule and a subring of $Q_{0}(R)$, then $C(A) \subseteq C(R)$ and $E(A) \subseteq E(R)$.

Proof. (1) Let $0 \neq x \in Q_{0}(R e)$; then $x: I \rightarrow R e$, where $I$ is an essential ideal of $R e$ and $x$ is a left $R e$-module homomorphism. Let $J$ be an essential ideal of $R$ such that $J e \subseteq R$. Then the mapping $\tilde{x}: J I \oplus \operatorname{Ann}_{R}(J I) \rightarrow R$ defined by $(a, b) \tilde{x}=a x$ is a nonzero element of $Q_{0}(R)$ satisfying $\tilde{x} e=\tilde{x}$. Thus $Q_{0}(R e) \subseteq Q_{0}(R) e$. For the reverse inclusion, choose $0 \neq x \in Q_{0}(R) e$. Then $K x \subseteq R$, for $K$ an essential ideal of $R$, and $e x=x e=x$. But then $K e$ is essential in $R e$, and $K e x=K x e \subseteq R e$. Thus $x$ restricted to $K e$ is an element of $Q_{0}(R e)$. (2), (3), and (4) are proved similarly.

(5) Let $c \in C(A)$; that is, $c: I \rightarrow A$ where $I$ is an essential ideal of $A$ and $c a=a c$, for all $a \in A$. Let $B=\{t \in I \cap R \mid t c \in A \cap R\}$. Then $B$ is an essential ideal of $A \cap R$, and $B c \neq 0$. Let $I^{\prime}=(A \cap R) B(A \cap R)$ and define $\tilde{c}: I^{\prime} \oplus \operatorname{Ann}_{R}\left(I^{\prime}\right) \rightarrow R$ by $(a, b) \tilde{c}=a c$. It is not difficult to show that $\tilde{c}$ is an $R-R$ bimodule homomorphism. Thus $\tilde{c} \in C(R)$, and so $C(A) \subseteq C(R)$.

We note here another easy fact about $Q_{0}(R)$ : if $A$ is any essential ideal of $R$ and $g \in$ Aut $R$ fixes $A$, then $g$ fixes $Q_{0}(R)$.

Now consider a finite group $G$ acting on $R$. We review some notation from [6]. For each $w \in G_{\text {inn }}$, we associate a subgroup $H_{w}$ as follows: $H_{w}$ is a subset of $G_{\text {inn }}$ maximal with respect to the property that $e_{w} \Pi_{h \in H_{w}} e_{h} \neq 0$. It is easy to see that $H_{w}$ must be a subgroup; we note also that if $w \neq 1, H_{w} \neq 1$, as $H_{w} \supseteq\langle w\rangle$. Let $f_{w}=\prod_{h \in H_{w}} e_{h}$, and let $S_{w}=\left\{g \in G \mid f_{w}^{g}=f_{w}\right\}$, the stabilizer of $f_{w}$ in $G$. Note that by the maximality of $H_{w}, e_{g} f_{w} \neq 0$ if and only if $g \in H_{w}$. Finally, let $X=\left\{1, g_{2}, \ldots, g_{k}\right\}$ be a set of right coset representatives of $S_{w}$ in $G$, and let $A_{w}$ be a $G$-stable essential ideal of $R$ such that $A_{w} f_{w} \subseteq R$.

Usually we shall fix some $w \in G_{\text {inn }}$, and for simplicity omit the subscript $w$ in the above.

LeMma 1.2. For $w \in G_{\text {inn }}$, let $H, f, S$, and $A$ be as above.

(1) In $R f, S_{\mathrm{inn}}=H$; moreover, the elements of $H$ become genuinely inner on $R N f$.

(2) If $f_{w}=1$ for some $w \in G_{\mathrm{inn}}$, then every element of $G_{\mathrm{inn}}$ becomes inner on $R N f$; in particular, $G_{\text {inn }}$ is a normal subgroup of $G$.

Proof. Clearly (2) follows from (1), as $S=G$. Thus it suffices to show (1). Choose $h \in S_{\text {inn }}$; that is, $h \in S$ is $X$-inner on $R f$. Now $A f$ is an $S$-invariant ideal of $R$, essential in $R f$, and $h$ is $X$-inner on $A f$. Since being $X$-inner on an ideal of $R$ implies $X$-inner on $R$ [7, Lemma 1.4], $h$ is $X$-inner on $R$. Thus $h \in G_{\text {inn }}$ and certainly $e_{h} f \neq 0$; this implies $h \in H$. Conversely, if $h \in H$, then $e_{h} f=f$; as $x_{h} x_{h}^{\prime}=e_{h}$, $\left(x_{h} f\right)\left(x_{h}^{\prime} f\right)=f$ and $x_{h} f \in R N f$. Thus each element of $H$ becomes genuinely inner on $R N f$. 
LEMMA 1.3. For $w, f, S$, and $H$ as above, the following are equivalent, for any $g \in G$ :

(1) $f=f^{g}$ (that is, $\left.g \in S\right)$,

(2) $\mathrm{ff}^{g} \neq 0$,

(3) $g \in N_{G}(H)$, the normalizer of $H$ in $G$,

(4) If $=(\text { If })^{g}$, for any $g$-stable ideal I such that If $\neq 0$.

Proof. We will show $(2) \Rightarrow(3) \Rightarrow(1) \Rightarrow(4) \Rightarrow(2)$. First observe that since $e_{h}^{g}=$ $e_{g^{-1} h g}$, we have $f^{g}=\prod_{h \in H} e_{g^{-1} h g}$. Thus if $f f^{g} \neq 0$, then $f e_{g^{-1} h g} \neq 0$ for all $h \in H$, which (by the maximality of $H$ ) implies $g^{-1} h g \in H$, all $h \in H$. Thus (2) $\Rightarrow$ (3). Also (3) $\Rightarrow$ (1), again using $e_{h}^{g}=e_{g^{-1} h g}$ and the definition of $f$. (1) $\Rightarrow(4)$ follows since $I$ is $g$-stable. Finally, (4) $\Rightarrow(2)$ since if $I f=(\text { If })^{g}$, there is some $a \in I, 0 \neq a f^{g} \in I f$, and so $a f^{g} f=a f^{g} \neq 0$, showing (2).

We now begin to construct our trace-like function. Recall that $X=\left\{1, g_{2}, \ldots, g_{k}\right\}$ is a set of right coset representatives for $S$ in $G$. Write $E=E(R)$ for simplicity. For $y f \in(R E f)^{S}=(R E)^{S} f$, define $t_{X}(y f)=\Sigma_{g \in X}(y f)^{g}$. We let $e=t_{X}(f)=\Sigma_{g \in X} f^{g}$.

Lemma 1.4. Let $X, E, f, t_{X}$, and e be as above. Then

(1) the set $\left\{f, f^{g_{2}}, \ldots, f^{g_{k}}\right\}$ consists of orthogonal idempotents,

(2) $0 \neq e \in E^{G}$, and $f^{g} e=f^{g}$ for each $g \in X$, consequently (RE)e is a G-stable ideal of $R E$,

(3) $t_{X}:(R E f)^{S} \rightarrow(R E)^{G} e$ is an $(R E)^{G}$-bimodule isomorphism.

Proof. (1) Say that $f^{g_{i}} f^{g_{j}} \neq 0$, for some $i, j$. Then $f^{g_{j} g_{i}^{-1}} \neq 0$, so by Lemma 1.3 , $g_{j} g_{i}^{-1} \in S$. But then $g_{j}$ and $g_{i}$ are in the same coset, giving $g_{i}=g_{j}$.

(2) Since $e=\Sigma_{g \in X} f^{g}$ and the $\left\{f^{g} \mid g \in X\right\}$ are orthogonal, $e f^{g}=f^{g}$, all $g \in X$. The fact that $e \in E^{G}$ will follow from the proof of (3).

(3) Since $G$ permutes the cosets of $S$, for each $g \in G$ we have $S g_{i} g=S g_{j}$. Since $y f \in(R E f)^{S}$, it follows that $(y f)^{g_{i} g}=(y f)^{g_{j}}$. But then

$$
t_{X}(y f)^{g}=\sum_{g_{i} \in X}(y f)^{g_{i} g}=\sum_{g_{j} \in X}(y f)^{g_{j}}=t_{X}(y f) ;
$$

thus $t_{X}(y f) \in(R E)^{G}$. Also, $t_{X}(y f) \in(R E)^{G} e$ since $f^{g} e=f^{g}$, all $g \in X$, and $t_{X}$ is injective by the orthogonality of the $\left\{f^{g_{i}}\right\}$. Finally, if $z e \in(R E)^{G} e$, then $z e=$ zet $_{X}(f)=t_{X}(z e f)=t_{X}(z f)$, hence $t_{X}$ is surjective.

To each $v \in G_{\text {inn }}$, we may associate various $H_{v}$. However, since $G$ is finite there exists $w \in G_{\text {inn }}$ with an $H_{w}$ such that $\left|H_{w}\right| \geqslant\left|H_{v}\right|$, for all $v \in G_{\text {inn. }}$. We call such a $w$ a maximal element of $G_{\text {inn }}$. Let $w$ be a maximal element and associate to it an $H_{w}$ of maximal order. We show that in this case, certain conjugates of $w$ give rise to the same $e$ as does $w$.

LEMMA 1.5. Let $w$ be a maximal element of $G_{\mathrm{inn}}$, and let $H, f, S, X$, and $e$ be as before. Choose $g \in X$. Then $v=g^{-1} w g \in G_{\text {inn }}$ with an $H_{v}=g^{-1} H g, f_{v}=f^{g}, S_{v}=$ $g^{-1} S g$, and $t_{X_{v}}\left(f^{g}\right)=e$.

Proof. Let $v=g^{-1} w g$. Then $0 \neq f^{g}=\prod_{h \in H} e_{g^{-1} h g}$, hence $H_{v} \supseteq g^{-1} H g$. However, by the maximality of the order of $H_{w}, H_{v}=g^{-1} H g$. Thus $f_{v}=f^{g}$, and it is easy to see 
that $S_{v}=g^{-1} S g$ and $X_{v}=g^{-1} X g$. Finally,

$$
t_{X_{v}}\left(f_{v}\right)=\sum_{x \in X}\left(f^{g}\right)^{g^{-1} x g}=\left(\sum_{x \in X} f^{x}\right)^{g}=e^{g}=e .
$$

We now specialize to the situation when $R$ has no nilpotent elements. We require here some results of Kharchenko. First of all, he proves in [11] that for any finite group $G$ acting on a ring $R$ with no nilpotent elements, $R^{G} \neq(0)$. Secondly, in [13] he studies the situation for $G$ acting on a semiprime ring $R$ such that the "algebra of the group" $B=\Sigma_{g \in G} \phi_{g}$ is semiprime. This is certainly true in our situation, as $B \subseteq R N$, which has no nilpotent elements. The specific results we use are [13, Theorem 4, Theorem 7, and Lemma 10]:

Proposition 1.6 (KhaRChenKo). Let $R$ be a ring with no nilpotent elements and $G$ be a finite group acting as automorphisms on $R$. Then

(a) the centralizer of $R^{G}$ in $Q_{0}(R)$ is $B$,

(b) $Q_{0}(R)^{G}=Q_{0}\left(R^{G}\right)$,

(c) if $I \in \mathcal{F}\left(R^{G}\right)$, then $R I$ contains some $K \in \mathcal{F}(R)$.

COROLlaRY 1.7. Let $R$ have no nilpotent elements, and $G$ be a finite group acting on $R$. Then $E\left(R^{G}\right)=E(R)^{G}$.

Proof. Choose $e \in E\left(R^{G}\right)$. Then by Proposition 1.6(b), $e \in Q_{0}(R)^{G}$. Moreover, since $e \in E\left(R^{G}\right)$, it centralizes $R^{G}$. Thus $e \in B$ by part (a) above. Hence $e \in R N$, which has no nilpotent elements. Since all idempotents in such rings are central, $e \in C(R)$; thus $e \in E(R)$, and also $e \in E(R)^{G}$ as it is fixed by $G$. Conversely, if $e \in E(R)^{G}$, then by part (b) above, $e \in Q_{0}\left(R^{G}\right)$. As $e$ centralizes $R$, we certainly have $e \in C\left(R^{G}\right)$, and so $e \in E\left(R^{G}\right)$.

The corollary is certainly false if $R$ has nilpotent elements, even if $|G|^{-1} \in R$, as can be seen by considering $R=M_{2}(\mathbf{Q})$ and $G=\langle g\rangle$, where $g$ is conjugation by

$$
\left(\begin{array}{rr}
1 & 0 \\
0 & -1
\end{array}\right)
$$

When $R$ has no nilpotent elements, then for any $x \in R$ we have $\{y \in R \mid y x=0\}$ $=\{y \in R \mid x y=0\}$. We denote this set by $\operatorname{Ann}_{R}(x)$.

LeMma 1.8. Let $R$ have no nilpotent elements, and let $I \neq 0$ be a $G$-stable right (left) ideal of $R$. Then $\operatorname{Ann}_{R}\left(I^{G}\right)=\operatorname{Ann}_{R}(I)$.

Proof. Let $I \neq 0$ be a $G$-stable right ideal of $R$. Then by [11, Theorem 2], $I^{G} \neq(0)$. Let $W=\operatorname{Ann}_{R}\left(I^{G}\right)$, a $G$-stable ideal of $R$. If $I W \neq 0$, then $(I W)^{G} \neq 0$. But then, $0 \neq(I W)^{G}(I W)^{G} \subseteq I^{G} I W=0$, a contradiction. Thus $I W=0$, so $W \subseteq$ $\operatorname{Ann}_{R}(I)$. The reverse inclusion is trivial.

2. Existence of trace-like functions for rings without nilpotent elements. Unless otherwise stated, we assume throughout this section that $R$ has no nilpotent elements, and prove the existence of essential trace-like functions on $R$. Our first lemma is known; we include it for the sake of completeness. 
LEMMA 2.1. Let $R$ be semiprime and $G$ a finite group acting on $R$ such that either (1) $R$ has no $|G|$-torsion, or (2) $G$ is $X$-outer on $R$, and consider the usual trace function $t_{G}(x)=\sum_{g \in G} x^{g}$. Then $t_{G}(R)$ is an essential ideal of $R^{G}$.

Proof. Case (1) follows from Bergman and Isaac's theorem [3] that $R^{G} \neq 0$ in this case. For then, $t(R) \supseteq t\left(R^{G}\right)=|G| R^{G} \neq 0$, and $|G| R^{G}$ is clearly essential in $R^{G}$.

Case (2) follows from the fact, proved in [15], that if $G$ is $X$-outer, then $t(\lambda) \neq 0$ for any nonzero left (right) ideal $\lambda$ of $R$. Thus for any $0 \neq a \in R^{G}, 0 \neq t(a R)=$ $a t(R) \subseteq a R^{G} \cap t(R)$. This proves that $t(R)$ is essential.

In order to prove the main theorem, we will actually prove a slightly more general result in which certain finite sets of central idempotents are adjoined to $R$. In $E=E(R)$, we let $E_{0}(R)=\{L \subset E$ such that $L$ is a finite multiplicatively-closed set, and $1=\sum t_{i}$ for some $\left.\left\{t_{i}\right\} \subset L\right\}$. Our aim is to prove

Proposition 2.2. Let $R$ be a ring without nilpotent elements and $G$ a finite group of automorphisms of $R$. Then there exist $L_{1}, L_{2}$ in $E_{0}(R)$ and an $R^{G}$-bimodule homomorphism $\tau: R L_{1} \rightarrow\left(R L_{2}\right)^{G}$, such that $\operatorname{Im} \tau$ is an essential $R^{G}$-submodule of $(R E)^{G}$.

We proceed by a series of reductions.

LEMMA 2.3. Let $e_{1}, e_{2} \in E^{G}$ with $e_{1}+e_{2}=1$, and let $R_{i}=R e_{i} \subseteq R E$, for $i=1,2$. If Proposition 2.2 is true for each $R_{i}$, then it is true for $R$.

Proof. We use Lemma 1.1. For, $E\left(R_{i}\right)=E(R) e_{i}$, and as $e_{i} \in E^{G}$, it is also true that $E_{0}\left(R_{i}\right) \subseteq E_{0}(R)$. Thus if we have $\tau_{i}: R_{i} L_{1}^{(i)} \rightarrow\left(R_{i} L_{2}^{(i)}\right)^{G}, i=1,2$, where $L_{j}^{(i)} \in$ $E_{0}\left(R_{i}\right)$, let $L_{1}=\left\langle L_{1}^{(1)}, L_{1}^{(2)}\right\rangle$, the subset of $E_{0}$ generated by $\left\{L_{1}^{(i)}\right\}$, and $L_{2}=$ $\left\langle L_{2}^{(1)}, L_{2}^{(2)}\right\rangle$. Then $R L_{1}=R_{1} L_{1}^{(1)} \oplus R_{2} L_{1}^{(2)}$, as the elements of $L_{1}^{(1)}$ and $L_{1}^{(2)}$ are orthogonal to each other. Thus we define $\tau=\tau_{1} \oplus \tau_{2}$ on $R L_{1}$; clearly $\tau\left(R L_{1}\right) \subseteq$ $\left(R L_{2}\right)^{G}$. Now $E=E e_{1} \oplus E e_{2}$; since $\tau_{i}\left(R_{i} L_{1}^{(i)}\right)$ is essential in $R_{i}\left(E e_{i}\right)$, for $i=1,2$, by assumption, it follows that $\operatorname{Im} \tau$ is essential in $R E=R_{1}\left(E e_{1}\right) \oplus R_{2}\left(E e_{2}\right)$.

COROllaRy 2.4. It suffices to prove Proposition 2.2 under the assumption that $p R=0$, where $p$ is a prime such that $p$ divides $|G|$.

Proof. Assume that $|G|=p_{1}^{n_{1}} \cdots p_{r}^{n_{r}}$, where the $p_{i}$ are distinct primes. For each $i=1, \ldots, r$, let $R_{p_{i}}=\left\{r \in R \mid p_{i}^{l} r=0\right.$, for some $\left.l\right\}$. Since $R$ has no nilpotent elements, $p_{i}^{l_{r}}=0$ gives $\left(p_{i} r\right)^{l}=0$, so $p_{i} r=0$; that is, $R_{p_{i}}=\left\{r \in R \mid p_{i} r=0\right\}$. Now each $R_{p_{i}}$ is a $G$-stable ideal of $R$; thus $I=\operatorname{Ann}_{R}\left(R_{1} \oplus \cdots \oplus R_{r}\right)$ is also a $G$-stable ideal, and $K=R_{1} \oplus \cdots \oplus R_{r} \oplus I$ is essential in $R$. We define $e_{1}, \ldots, e_{r}, e_{0} \in E^{G}$ as follows: for $i=1, \ldots, r, e_{i}: K \rightarrow R$ via $e_{i}\left(a_{1}, \ldots, a_{r}, b\right)=a_{i}$, and $e_{0}: K \rightarrow R$ via $e_{0}\left(a_{1}, \ldots, a_{r}, b\right)=b$. It is not difficult to check that the $e_{i} \in E^{G}$, and moreover $e_{1}+\cdots+e_{r}+e_{0}=1$. As the $e_{i}$ are orthogonal, the set $L=\left\{e_{1}, \ldots, e_{r}, e_{0}\right\}$ is contained in $E_{0}(R)$. Let $R_{i}=R e_{i}, i=0, \ldots, r$; it is not difficult to see that $p_{i} R_{i}=(0), i=1, \ldots, r$, and that $R_{0}$ is $|G|$-torsion-free. By Lemma 2.3 (iterated), it suffices to prove Proposition 2.2 for each $R_{i}, i=0, \ldots, r$. But by Lemma 2.1, since $R_{0}$ has no $|G|$-torsion, the proposition is true for $R_{0}: t_{G}\left(R_{0}\right)$ is essential in $R_{0}^{G}$. Thus it suffices to prove the the proposition for $R_{i}, i=1, \ldots, r$; this proves the corollary. 
Lemma 2.5. Proposition 2.2 is true if $|G|=p$, a prime.

Proof. By Corollary 2.4, we may assume that $p R=(0)$. We claim it suffices to prove the proposition under the assumption that $R^{G}$ contains no nonzero ideals of $R$. For, say that such ideals exist, and let $I$ be an ideal of $R$ maximal with respect to the property that $I \subseteq R^{G}$. Letting $J=\operatorname{Ann}_{R}(I), J$ is a $G$-stable ideal of $R$ and $K=I \oplus J$ is essential in $R$. As in the proof of Corollary 2.4, we have $e_{1}, e_{2} \in E^{G}$ given by $e_{i}: K \rightarrow R$, where $e_{1}(a, b)=a$ and $e_{2}(a, b)=b$. Let $R_{1}=R e_{1}$ and $R_{2}=$ $R e_{2}$. Now $R e_{1}$ is fixed by $G$, since $I$ is an essential ideal of $R e_{1}$ and $I$ is fixed. Thus, letting $\tau_{1}$ be the identity function on $R e_{1}$, we see that Proposition 2.2 is true for $R e_{1}$. Now $R e_{2}$ has the property that $\left(R e_{2}\right)^{G}$ contains no nonzero ideals $W$ of $R e_{2}$. For if such a $W$ existed, $W \cap J \neq 0$, as $J$ is essential in $\operatorname{Re}_{2}$, and then $I \oplus W \cap J \subseteq R^{G}$, which contradicts our choice of $I$. By Lemma 2.3, it suffices to show that Proposition 2.2 is true for $R e_{2}$, proving the claim.

We now use another result of Bergman and Isaacs [3, Proposition 3.3] which says that if $|G|=p, G$ acts faithfully on $R$, and $p R=0$, then $t_{G}(R) \neq 0$. We will show that, under our assumption, $t_{G}(R)$ is actually essential in $R^{G}$. For, say that for some $a \in R^{G}, 0=a t_{G}(R)=t_{G}(a R)$. By the result just mentioned, $G$ must act trivially on $a R$; that is, $a R \subseteq R^{G}$. But then $t_{G}(R a R)=t_{G}(R) a R=(0)$. Thus $R a R \subseteq R^{G}$. As we are assuming that $R^{G}$ contains no nonzero ideals of $R^{G}$, we must have $R a R=0$, and so $a=0$. Thus $t_{G}(R)$ is essential in $R^{G}$, proving the lemma.

LeMma 2.6. Assume that $p R=0$, for some prime $p$, and that $G$ is a simple group of automorphisms which become genuinely inner on $Q_{0}(R)$. Then Proposition 2.2 is true for $G$ acting on $R$.

Proof. We apply a theorem of Faith-Kharchenko [16, Theorem 11] which says that under the hypotheses of the lemma, $p$ does not divide the order of $G^{\prime}$, the commutator subgroup. As $G$ is simple, either $G=G^{\prime}$ (in which case we may use $t_{G^{\prime}}$ by Lemma 2.1 ), or $G^{\prime}=\langle 1\rangle$. In the second case, as $G$ is simple and abelian, $|G|=q$, for a prime $q$. We are now done by applying Lemma 2.5 .

The next lemma is needed in order to do induction.

Lemma 2.7. Assume that Proposition 2.2 is true for all groups $H$ such that $|H|<n$, for some $n$, where $H$ may act on any ring without nilpotent elements. If $G$ is a group with $|G|=n$ which has a proper normal subgroup $M$, then Proposition 2.2 is also true for any action of $G$.

Proof. Let $G$ act on a given ring $R$ with no nilpotent elements. Since $|M|<n$, by our assumption there exist $L_{1}, L_{2} \in E_{0}(R)$ and an $R^{M}$-bimodule homomorphism $\tau_{1}: R L_{1} \rightarrow\left(R L_{2}\right)^{M}$ such that $\operatorname{Im} \tau_{1}$ is essential in $(R E)^{M}$. Now let $L_{2}^{\prime}=\left\langle e^{g}\right| e \in$ $\left.L_{2}, g \in G\right\rangle$, the $G$-stable subset of $E_{0}(R)$ generated by $L_{2}$. Since $\left(R L_{2}^{\prime}\right)^{M}$ is a $G / M$-stable subring of $R E$, and $|G / M|<|G|$, by our assumption there exist $L_{3}, L_{4} \in E_{0}\left(\left(R L_{2}^{\prime}\right)^{M}\right)$ and an $\left(\left(R L_{2}^{\prime}\right)^{M}\right)^{G / M}$-bimodule homomorphism $\tau_{2}:\left(R L_{2}^{\prime}\right)^{M} L_{3}$ $\rightarrow\left(\left(R L_{2}^{\prime}\right)^{M} L_{4}\right)^{G / M}$ such that $\operatorname{Im} \tau_{2}$ is essential in $\left(\left(R L_{2}^{\prime}\right)^{M} E\left(\left(R L_{2}^{\prime}\right)^{M}\right)\right)^{G / M}$. 
We first simplify some of the above expressions. By Corollary 1.7 and Lemma 1.1, $E\left(\left(R L_{2}^{\prime}\right)^{M}\right)=\left(E\left(R L_{2}^{\prime}\right)\right)^{M}=E(R)^{M}$. Also, for any $G$-stable subset $S,\left(S^{M}\right)^{G / M}=$ $S^{G}$. Thus, in the above, $L_{3}$ and $L_{4}$ are in $E_{0}(R)^{M}$ and $\tau_{2}$ is an $\left(R L_{2}^{\prime}\right)^{G}$-bimodule homomorphism such that $\operatorname{Im} \tau_{2}$ is essential in $\left(\left(R L_{2}^{\prime}\right)^{M} E(R)^{M}\right)^{G}$. It is therefore clear at least that $\tau=\tau_{2} \circ \tau_{1}$ is an $R^{G}$-bimodule homomorphism. We will show that $\operatorname{Im} \tau_{2} \circ \tau_{1}$ is an essential $R^{G}$-submodule of $(R E)^{G}$.

Now $R^{G}$ is an essential $R^{G}$-submodule of $(R E)^{G}$ by Lemma 1.8 (if $a R^{G}=0$, for $a \in(R e)^{G}$, then $a R=0$, and so $a=0$ in $\left.R E\right)$. Thus $\left(\left(R L_{2}^{\prime}\right)^{M} E(R)^{M}\right)^{G}$ is an essential $R^{G}$-submodule of $(R E)^{G}$, as it contains $R^{G}$, and thus $\operatorname{Im} \tau_{2}$ is an essential $R^{G}$-submodule of $(R E)^{G}$. As $\operatorname{Im} \tau_{1}$ is essential in $(R E)^{M},\left(\operatorname{Im} \tau_{1}\right)^{G}$ is essential in $(R E)^{G}$, by Lemma 1.8 again using $G / M$ as the group. It follows that $W=$ $\left(\operatorname{Im} \tau_{1}\right)^{G} \cap R^{G}$ is essential in $R^{G}$.

Let $L=\left\langle L_{1}, L_{2}^{\prime}, L_{3}, L_{4}\right\rangle \subseteq E_{0}(R)$. As $L$ is a finite set, there exists an ideal $I \in \mathscr{F}(R)$ such that $I L \subseteq R$. Again by Lemma $1.8, I^{G}$ is essential in $R^{G}$. Letting $U=W I^{G}\left(\operatorname{Im} \tau_{2}\right)$, it follows from the above remarks that $U$ is an essential $R^{G}$-submodule of $(R E)^{G}$. Moreover,

$$
\begin{aligned}
U & =W I^{G} \tau_{2}\left(\left(R L_{2}^{\prime}\right)^{M} L_{3}\right)=\tau_{2}\left(W I^{G}\left(R L_{2}^{\prime}\right)^{M} L_{3}\right) \subseteq \tau_{2}\left(W I^{G}(R L)^{M}\right) \\
& \subseteq \tau_{2}\left(W(I R L)^{M}\right) \subseteq \tau_{2}\left(W R^{M}\right) \subseteq \tau_{2}\left(\left(\operatorname{Im} \tau_{1}\right) R^{M}\right) \subseteq \tau_{2}\left(\operatorname{Im} \tau_{1}\right)=\operatorname{Im} \tau_{2} \circ \tau_{1} .
\end{aligned}
$$

This shows that $\operatorname{Im} \tau_{2} \circ \tau_{1}$ is an essential $R^{G}$-submodule of $(R E)^{G}$.

Finally,

$$
\tau_{2} \circ \tau_{1}\left(R L_{1}\right) \subseteq \tau_{2}\left(R L_{2}\right)^{M} \subseteq\left(\left(R L_{2}^{\prime}\right)^{M} L_{4}\right)^{G / M} \subseteq\left((R L)^{M}\right)^{G / M} \subseteq(R L)^{G} .
$$

Thus $\tau_{2} \circ \tau_{1}: R L_{1} \rightarrow(R L)^{G}$. Using $\tau=\tau_{2} \circ \tau_{1}$, Proposition 2.2 is true for $G$, proving the lemma.

Proof of Proposition 2.2. We proceed by induction on $|G|$. By Lemma 2.7, we may assume that $G$ is simple. If $G$ is $X$-outer on $R$, we are done by Lemma 2.1, so we may assume that $G_{\mathrm{inn}} \neq\langle 1\rangle$. We consider two cases.

Case (1). There exists $1 \neq w \in G_{\text {inn }}$ with $f_{w} \in E^{G}$ (notation as in $\S 1$ ). By Lemmas 1.2 and 1.3, $H_{w}$ is normal in $G$ and its elements become genuinely inner in $(R N) f_{w}$. Since $w \neq 1, H_{w} \neq 1$ (this is easy to verify) and thus $H_{w}=G$ as $G$ is simple. Write $e=f_{w}$ for simplicity. Now as $Q_{0}(R e)=Q_{0}(R) e$, the elements of $G$ are inner on $Q_{0}(R e)$. We may thus apply Lemma 2.6 and conclude that Proposition 2.2 is true on $R e$. If $e=1$, we are done.

Case (2). For all $1 \neq w \in G_{\text {inn }}, f_{w} \notin E^{G}$. We choose $w$ to be a maximal element of $G_{\text {inn }}$. Let $H, f, S, X$, and $e$ be related to $w$ as in $\S 1$. As $S$ is the stabilizer of $f_{w} \notin E^{G}$, $|S|<|G|$. Apply induction to the ring $R f$ and $S$ to find $L_{1}, L_{2} \in E_{0}(R f)=E_{0}(R) f$ and an $R^{S}$-bimodule homomorphism $\tau:(R f) L_{1} \rightarrow\left((R f) L_{2}\right)^{S}$ such that $\operatorname{Im} \tau$ is essential in $(R E f)^{S}$. We next apply Lemma 1.4: $t_{X}:(R E f)^{S} \rightarrow(R E)^{G} e$ is an $(R E)^{G}$ bimodule isomorphism, where $t_{X}(y f)=\Sigma_{g \in X}(y f)^{g}$ and $e=t_{X}(f)$. Let $L_{2}^{\prime}=$ $\left\langle L_{2}^{g}, f^{g} \mid g \in X\right\rangle$. Since $f^{g} e=f^{g}$, all $g \in X$ (by Lemma 1.4), $E_{0}\left(R f^{g}\right)=E_{0}(R) f^{g} \subseteq$ $E_{0}(R) e=E_{0}(R e)$, all $g \in X$, and thus $L_{2}^{\prime} \in E_{0}(R e)$. It is not difficult to see that 
$t_{X}\left(\left(R L_{2} f\right)^{S}\right) \subseteq\left(R L_{2}^{\prime}\right) e$. As $t_{X}\left(\left(R L_{2} f\right)^{S}\right) \subseteq(R E)^{G} e$, it follows that $t_{X}\left(\left(R L_{2} f\right)^{S}\right)$ $\subseteq\left(R L_{2}^{\prime}\right)^{G} e$, and so $\mu_{1}=t_{X} \tau:(R f) L_{1} \rightarrow\left(R L_{2}^{\prime}\right)^{G} e$. Moreover, $\operatorname{Im} \mu_{1}$ is essential in $(R E)^{G} e$, since $\operatorname{Im} \tau$ is essential in $(R E f)^{S}$ and $t_{X}$ is an $(R E)^{G}$-bimodule isomorphism.

Now for any $g \neq 1$ in $X$, let $v=g^{-1} w g$. Then $f_{v}=f^{g}$ and $t_{X_{v}}\left(f^{g}\right)=e$ by Lemma 1.5. Repeating the previous argument, there exist $L_{1, g} \in E_{0}\left(R f^{g}\right)$ and $L_{2, g}^{\prime} \in E_{0}(R e)$ and an $R^{G}$-bimodule homomorphism $\mu_{g}:\left(R f^{g}\right) L_{1, g} \rightarrow\left(R L_{2, g}^{\prime}\right)^{G} e$ such that $\operatorname{Im} \mu_{g}$ is essential in $(R E)^{G} e$. Let $L_{3}=\left\langle L_{1, g} \mid g \in X\right\rangle \subseteq E_{0}(R)$, and note that since the $\left\{f^{g}\right\}$ are orthogonal, $(R e) L_{3}=\Sigma_{g \in X} \oplus\left(R f^{g}\right) L_{1, g}$. Letting $L_{4}=\left\langle L_{2, g}^{\prime} \mid g \in X\right\rangle \in E_{0}(R e)$, we define $\mu$ on $(R e) L_{3}$ by $\mu=\Sigma_{g \in G} \oplus \mu_{g}$ and see that $\mu:(R e) L_{3} \rightarrow\left(R L_{4}\right)^{G} e$ is an $R^{G}$-bimodule homomorphism such that $\operatorname{Im} \mu$ is essential in $(R E)^{G} e$. If $e=1$, we are done.

We may therefore assume that $e \neq 1$ in both Cases (1) and (2). Consider $1-e$ and $R(1-e)$. By Lemma 2.3, it would suffice to prove Proposition 2.2 on the ring $R(1-e)$. We therefore repeat the argument from the beginning on $R(1-e)$. If $G$ is $X$-outer on $R(1-e)$ we would be done, so $G_{\text {inn }} \neq 1$ on $R(1-e)$. Therefore, repeating Case (1) or (2), in either case we have $1 \neq w_{1} \in G_{\text {inn }}$ and $H_{w_{1}}$ as before. We get $e_{1} \neq 0\left(e_{1}=f_{w_{1}}\right.$ in Case (1), and $e_{1}=t_{X_{1}}\left(f_{w_{1}}\right)$ in Case (2)) and Proposition 2.2 is true on $\operatorname{Re}_{1}$. The crucial point is that $H_{w_{1}} \neq H_{w}$. For, $0 \neq f_{w_{1}}=\Pi_{h \in H_{w_{1}}} \tilde{e}_{h}$, where $\tilde{e}_{h}=e_{h}(1-e)$, the "restriction" to $R(1-e)$. If $H_{w_{1}}=H_{w}$, then $f_{w_{1}}=$ $\Pi_{h \in H_{w}} \tilde{e}_{h}=\tilde{f}_{w}=f_{w}(1-e)=0$, a contradiction. Thus $H_{w_{1}} \neq H_{w}$.

Iterating the procedure, at the $i$ th-stage we get $e_{i} \in R\left(1-e-e_{1}-\cdots-e_{i-1}\right)$ and $H_{w_{i}} \neq H_{w_{j}}$ for all $j=0, \ldots, i-1$. Since the number of distinct subsets of $G$ is finite, the process must terminate. This proves Proposition 2.2.

Our main theorem follows almost immediately.

THEOREM 2.8. Let $R$ be a ring with no nilpotent elements and $G$ a finite group acting as automorphisms on $R$. Then there exists a finite subset $L$ of $E=E(R)$ and an $R^{G}$-bimodule homomorphism $\tau: R \rightarrow(R L)^{G}$ such that $\tau(R)$ is essential in $(R E)^{G}$. Moreover, for some essential ideal $K$ of $R, \tau(K) \subseteq R^{G}$ and is essential in $R^{G}$.

Proof. By Proposition 2.2 there exist $L_{1}, L_{2} \in E_{0}(R)$ and $\tau: R L_{1} \rightarrow\left(R L_{2}\right)^{G}$ so that $\operatorname{Im} \tau$ is essential in $(R E)^{G}$. Now, since $L_{1}$ and $L_{2}$ are finite, there exists $I \in \mathscr{F}(R)$ such that $I\left(L_{1} \cup L_{2}\right) \subseteq R$. Then $I^{G}$ is essential in $R^{G}$ (by Lemma 1.8), so $\tau\left(I^{G} R L_{1}\right)=I^{G} \tau\left(R L_{1}\right) \subseteq R^{G}$ and is essential in $R^{G}$. By Proposition 1.6, part (c), $I^{G} R$ contains some $K \in \mathscr{F}(R) . \tau(K) \subseteq R^{G}$ since $\tau\left(I^{G} R\right) \subseteq R^{G}$, and it is easy to see that $\tau(K)$ is essential in $R^{G}$.

COROLlaRY 2.9. If $R$ is a ring with no nilpotent elements such that $E(R) \subseteq R$, then for any $G$ acting as automorphisms of $R$, there exists an $R^{G}$-bimodule homomorphism $\tau: R \rightarrow R^{G}$ such that $\tau(R)$ is essential in $R^{G}$.

We note that such " $E$-closed" rings can be constructed from $R$ itself. For, by a result of Martindale, the central closure $R C$ of $R$ is centrally closed (that is, the extended center of $R C$ is just $C$ ). Thus $R C$ is $E$-closed, and moreover, $R E(R)$ is $E$-closed since $E(R E(R))=E(R)$. 
REMARK 2.10. Assume we are given a $\tau$ as in Theorem 2.8, and let $C_{0}$ be any subring of $C^{G}$. Then $\tau$ may be extended to an $\left(R C_{0}\right)^{G}$-bimodule homomorphism $\tau_{1}: R C_{0} \rightarrow\left(R C_{0} L\right)^{G}$.

Proof. If $x \in R C_{0}$, write $x=\Sigma_{i} r_{i} c_{i}, r_{i} \in R, 0 \neq c_{i} \in C_{0}$. Define $\tau_{1}(x)=$ $\Sigma_{i} \tau\left(r_{i}\right) c_{i} \cdot \tau_{i}$ will certainly have the desired properties; it suffices to show it is well defined. That is, say that $x=0$; we wish to show $\tau_{1}(x)=0$. Since $c_{i} \in C^{G} \subseteq C\left(R^{G}\right)$ and $R^{G}$ is semiprime, we may choose $I \in \mathscr{F}\left(R^{G}\right)$ such that $0 \neq c_{i} I \subseteq R^{G}$, all $i$. Thus for any $a \in I, x a \in R$, and $\tau$ is defined on $x a$. Thus $0=x a=\tau(x a)=\Sigma_{i} \tau\left(r_{i} c_{i} a\right)$ $=\Sigma_{i} \tau\left(r_{i}\right) c_{i} a=\tau_{1}(x) a$. Since $\tau_{1}(x) I=0$, it follows that $\tau_{1}(x)=0$.

As noted in the introduction, when $R$ is a domain, we obtain the known result on partial trace functions.

COROllaRY 2.11 [16]. Let $R$ be a domain, and $G$ a finite group acting as automorphisms of $R$. Then there exists $\Lambda \subseteq G$ so that $0 \neq t_{\Lambda}(R) \subseteq R^{G}$.

Proof. Since $E(R)=\{0,1\}$, Lemma 2.3 is not needed, and Case (2) of the proof of the Proposition 2.2 cannot arise as $1 \in E^{G}$. Thus, we must only use induction (noting that the composition of two partial trace functions is another partial trace function) and note that for $G$ simple, we either use $\tau=t_{G}$ or $\tau=\mathrm{id}$, depending on whether $G$ acts faithfully or trivially (Lemmas 2.5 and 2.6). This is in fact the argument given in [16].

REMARK 2.12. The proof of Proposition 2.2 actually shows the following: let a simple group $G$ act on a ring $R$ with no nilpotent elements. Then there exists a finite set of orthogonal idempotents $e_{1}, \ldots, e_{n} \in E^{G}$, with $e_{1}+\cdots+e_{n}=1$, such that $G$ is $X$-outer on $R e_{1}$, every element of $G$ becomes genuinely inner on $Q_{0}\left(R e_{2}\right)$, and on each of $R e=R e_{3}, \ldots, R e_{n}$, we have the situation occurring in Case (2) of the proof of Proposition 2.2. That is, for all $1 \neq w \in G_{\text {inn }}, f_{w} \notin E^{G}$, so choosing $w$ to be a maximal element of $G_{\text {inn }}, S$ the stabilizer of $f_{w}$, and $X$ a set of right coset representatives of $S$ in $G, e=\Sigma_{g \in X} f_{w}^{g}$. Moreover, if $H_{i}$ is the $H_{w}$ we get for $G$ acting on $R e_{i}, i=3, \ldots, n$, then $H_{i} \neq H_{j}$ if $i \neq j$.

It is also clear from our construction of $\tau$, and the various reductions in the proof of the theorem, that Case (2) in Proposition 2.2 is the most interesting (as in all other cases, $\tau$ comes from adding together traces or the identity function). We therefore illustrate the procedure with an example.

EXAMPLE 2.13. We use Example 1.1 of [7]; it is shown in [7] that $t_{G}(R) \equiv 0$ in this example. Let $G$ be any nonabelian simple group and let $H$ be a proper subgroup. Say $[G: H]=n$ and consider $G$ as a group of permutations of the $n$ right cosets of $H$ in $G$, say $\left\{H, H a_{2}, \ldots, H a_{n}\right\}$. Choose any prime $p$ dividing $|H|$, let $F$ be a field of characteristic $p$, and let $R=\sum_{i=1}^{n} \oplus(F)_{i}$, the direct sum of $n$ copies of $F$. We let $G$ act on $R$ by permuting the $n$ summands as it permutes the cosets. Note that $H$ itself is the stabilizer of $(F)_{1}$, the stabilizer of $(F)_{i}$ is $H_{i}=a_{i}^{-1} H a_{i}$, and so $G_{\text {inn }}=\cup_{i} H_{i}$. Let $e_{i}=\left(0, \ldots, 1_{i}, \ldots, 0\right) \in E(R), i=1, \ldots, n$; clearly $E(R)$ consists of finite sums of the $\left\{e_{i}\right\}$ and 0 .

Choose any $1 \neq w \in H \subseteq G_{\text {inn }}$, and say that $w$ lies in $k$ of the $H_{i}$. By reordering the cosets, we may assume that $w \in H_{1} \cap \cdots \cap H_{k}$, and so $e_{w}=e_{1}+\cdots+e_{k}$. By 
definition, $H_{w}$ is a subgroup of $G_{\text {inn }}$ maximal with respect to the property that $e_{w} \Pi_{h \in H_{w}} e_{h} \neq 0$. We claim that $H_{w}=H_{i}$, for any $i=1, \ldots, k$. For, given $H_{w}$, let $f_{w}=\prod_{h \in H_{w}} e_{h}$; as $e_{w} f_{w} \neq 0, e_{i} f_{w} \neq 0$ for some $i, 1 \leqslant i \leqslant k$. Then $e_{i} e_{h} \neq 0$, all $h \in H_{w}$, and so $e_{i} e_{h}=e_{i}$. It follows that $h$ stabilizes $e_{i}$, and so $(F)_{i}$; that is, $h \in H_{i}$, so $H_{w} \subseteq H_{i}$. But for all $h \in H_{i}, e_{i} e_{h}=e_{i}$; thus $H_{w}=H_{i}$ by the maximality of $H_{w}$. Given $H_{i}$, any $i=1, \ldots, k$, clearly $e_{w} \Pi_{h \in H_{i}} e_{h} \neq 0$, so $H_{i} \subseteq$ some $H_{w}$. By the above argument, $\left|H_{w}\right|=\left|H_{i}\right|$ and so $H_{i}=H_{w}$. Thus we have $k$ choices for $H_{w}$. For ease in notation, we use $H_{w}=H_{1}=H$.

Then $e_{1} f_{w}=e_{1}$, so $f_{w}$ is a sum of $\left\{e_{i}\right\}$ including $e_{1}$. Again renumbering the cosets, assume that $f_{w}=e_{1}+\cdots+e_{m}$, for some $m$. From $\S 1$, the stabilizer $S$ of $f_{w}$ is also equal to $N_{G}(H)$, the normalizer of $H$ in $G$. We claim that $S=H \cup H a_{1} \cup \cdots \cup H a_{m}$. For, as $G$ is a union of $\left\{H a_{j}\right\}$, we only need to consider the coset representatives $\left\{a_{j}\right\}$. If $a_{j} \in S$, then $a_{j}^{-1} H a_{j}=H$, so every $h \in H$ stabilizes $(F)_{j}$, so $e_{h} e_{j}=e_{j}$, all $h \in H$, so $f_{w} e_{j}=e_{j}$, using $H=H_{w}$. As the $\left\{e_{i}\right\}$ are central orthogonal idempotents, it follows that $e_{j} \in\left\{e_{1}, \ldots, e_{m}\right\}$, so $1 \leqslant j \leqslant m$. Conversely, if $1 \leqslant j \leqslant m$, then $f_{w} e_{j}=e_{j}$, and $e_{h} e_{j}=e_{j}$, all $h \in H=H_{w}$. Thus $h$ stabilizes $(F)_{j}$, so $h \in H_{j}$. That is, $H \subseteq H_{j}$, so $H=H_{j}=a_{j}^{-1} H a_{j}$. Thus $a_{j} \in S$. This proves the claim.

We now consider $S$ acting on $R f_{w}=\sum_{j=1}^{m} \oplus(F)_{j}$, where $S$ acts (as did $G$ ) by permuting the summands as if they were the cosets $\left\{H, H a_{2}, \ldots, H a_{m}\right\}$. Now $H$ is normal in $S$, so acts as the identity permutation; $S$ acts as right multiplication by $S / H$. But $S / H \cong\left\{\overline{1}, \bar{a}_{2}, \ldots, \bar{a}_{m}\right\}$, so this is just the regular representation of $S / H$ on itself, and $m=|S / H|$. It is not difficult to see that the trace function $t_{S / H}: R f_{w}$ $\rightarrow\left(R f_{w}\right)^{S}$ is given by $t_{S / H}\left(c_{1}, \ldots, c_{m}\right)=\left(\sum_{j=1}^{m} c_{j}, \ldots, \sum_{j=1}^{m} c_{j}\right)$. Thus $t_{S / H}$ is onto $\left(R f_{w}\right)^{S}$, and we may use $\tau=t_{S / H}$. (In the proof of Proposition 2.2, we used induction at this point since $|S|<|G|$.)

Now let $X=\left\{1, b_{2}, \ldots, b_{q}\right\}$ be a set of coset representatives of $S$ in $G$; we may assume $\left\{b_{r}\right\} \subseteq\left\{a_{i}\right\}$. Then $e=t_{X}\left(f_{w}\right)=\Sigma_{r} f_{w}^{b_{r}}=1$, and $t_{X}:\left(R f_{w}\right)^{S} \rightarrow R^{G}$ (an isomorphism) is just the mapping which takes the $m$-tuple $(c, c, \ldots, c) \in\left(R f_{w}\right)^{S}$ to the $n$-tuple $(c, \ldots, c) \in R^{G}$. Then $\mu_{1}=t_{X} \circ \tau: R f_{w} \rightarrow R^{G}$ is given by $\mu_{1}\left(c_{1}, \ldots, c_{m}\right)=$ $\left(\sum_{j=1}^{m} c_{j}, \ldots, \sum_{j=1}^{m} c_{j}\right)$, an $n$-tuple.

Repeat this for $v_{r}=b_{r}^{-1} w b_{r}, f_{r}=f_{w}^{b_{r}}, S_{r}=b_{r}^{-1} H b_{r}$, to obtain $\mu_{r}: R f_{r} \rightarrow R^{G}$ as above. As $R=\sum_{r=1}^{q} \oplus R f_{r}$, we get $\mu=\sum_{r=1}^{q} \oplus \mu_{r}: R \rightarrow R^{G}$ given by $\mu\left(d_{1}, \ldots, d_{n}\right)=$ $\left(\sum_{i=1}^{n} d_{i}, \ldots, \sum_{i=1}^{n} d_{i}\right) \in R^{G}$, the desired function.

As computed in [7], $t_{G}\left(d_{1}, \ldots, d_{n}\right)=|H| \mu\left(d_{1}, \ldots, d_{n}\right)$; thus when $F$ has characteristic $p$ and $p|| H \mid, t_{G}(R) \equiv 0$.

3. Application to affine fixed rings. The main result of this section is Theorem 3.3, stated in the introduction; in it we use the existence of the trace function constructed in $\$ 2$ to show that, under certain hypotheses, the fixed subring of an affine ring is also affine. This result extends the result of [17, Theorem 2], where the analogous result was proved for domains.

The proof of [17, Theorem 2] used the so-called "trace ring" for a prime $P I$ ring $R$, used in [20 and 2]. That is, consider $R$ as an order in $M_{n}(D)$, where $D$ is a division 
ring with center $Z$; for $K$ a maximal subfield of $D$, we have $R \hookrightarrow M_{n}(D) \hookrightarrow M_{n}(D)$ $\otimes_{Z} K \cong M_{m}(K)$. The trace ring $T=T(R)$ is the subring of $M_{m}(K)$ generated by the coefficients of the characteristic polynomials of all $r \otimes 1 \in M_{m}(K), r \in R$. It is known that $T \subseteq M_{n}(D)$; thus $T \subseteq Z$ so is a domain. If $R$ is an algebra over a commutative ring $A, T$ is chosen to be the $A$-algebra generated by the above elements.

In order to extend [17, Theorem 2], we first must extend the notion of trace ring to the case of semiprime Noetherian PI rings. We wish to thank L. W. Small, who suggested to us the appropriate definition in this case, and who also suggested the arguments in Proposition 3.1 below.

Thus, let $R$ be a semiprime Noetherian $P I$ ring. $R$ has a semisimple Artinian classical quotent ring $Q(R)=Q_{1} \oplus \cdots \oplus Q_{r}$, where the $Q_{i}$ are simple rings. Let $E=\left\{e_{1}, \ldots, e_{r}\right\}$ be the set of primitive central idempotents of $Q(R)$, and let $R_{i}=R e_{i}$, for each $i$. Note that $Q\left(R_{i}\right)=Q_{i}$. Let $T_{i}=T\left(R_{i}\right)$, the trace ring of the prime $P I$ ring $R_{i}$ as above. We now define the trace ring of $R$ to be $\tilde{T}=\sum_{i=1}^{r} \oplus T_{i}$.

Note that $e_{i} \in T_{i}$, all $i$, by construction, and so letting $R^{\prime}=\Sigma_{i} R_{i}=\Sigma_{i} \oplus R e_{i}$, we have $\tilde{T} R=\tilde{T} R^{\prime}$, the subring of $Q(R)$ generated by $\tilde{T}$ and $R$. The rings $\tilde{T}$ and $\tilde{T} R$ behave very much like their counterparts in the prime case; all of the properties listed in the next proposition are known to be true when $R$ is prime [17, Proposition 3].

Proposition 3.1. Let $R$ be a semiprime Noetherian PI ring, with trace ring $\tilde{T}$ as above. Then:

(1) $\tilde{T} R$ is integral aver $\tilde{T}$,

(2) $\tilde{T} R$ is a finite $R$-module (and so is Noetherian),

(3) if $R$ is also an affine algebra over a commutative Noetherian ring $A$, then $\tilde{T}$ is affine over $A$, and $\tilde{T} R$ is a finite $\tilde{T}$-module.

Proof. (1) Choose $r \in \tilde{T} R^{\prime}$; then $r=\sum r_{i}$, where $r_{i} \in R_{i} T_{i}$. Now $r_{i}$ is integral over $T_{i}$ by construction. Thus each $r_{i}$ is integral over $\tilde{T}$, and so $r$ is integral over $\tilde{T}$ as it is a sum of commuting integral elements.

(2) By the result for prime rings (actually due to Schelter [20]), $T_{i} R_{i}$ is finite over $R_{i}$, for all $i$. Thus $T_{i} R_{i}$ is finite over $R^{\prime}$, and so $\tilde{T} R=\Sigma T_{i} R_{i}$ is finite over $R^{\prime}$. Since $R^{\prime}$ is a finite $R$-module, $\tilde{T} R$ is finite over $R$.

(3) As remarked earlier, when $R$ is an algebra, $\tilde{T}$ is also an algebra. Now each $T_{i}$ is affine over $A$, by the prime case, and so $\tilde{T}=\Sigma T_{i}$ is affine over $A$. Similarly $T_{i} R_{i}$ is finite over $T_{i}$, all $i$, by the prime case. Thus each $T_{i} R_{i}$, and so $\tilde{T} R=\tilde{T} R^{\prime}=\sum T_{i} R_{i}$, is finite over $\tilde{T}$.

We now consider the action of a finite group $G$ on $R$. $G$ has a unique extension to $Q(R)$, and so permutes the set $E$ of central idempotents. Thus for any $g \in G$, $R_{i}^{g}=R_{j}$ for some $j$; moreover $Z_{i}^{g}=Z_{j}$, where $Z_{i}$ is the center of $Q\left(R_{i}\right)$. It follows that $T_{i}^{g}=T_{j}$, and thus the trace ring $\tilde{T}=\sum T_{i}$ is $G$-stable. Now if $\tilde{T}$ is an affine $A$-algebra, then $\tilde{T}$ is a finite $\tilde{T}^{G}$-module and $\tilde{T}^{G}$ is affine, by the classical results of $\mathrm{E}$. Noether for commutative rings. 
Finally, we are able to show the relationship of trace functions to affine algebras.

Proposition 3.2. Let $R$ be a semiprime Noetherian PI ring which is an affine algebra over a commutative Noetherian ring $A$. Let $G$ be a finite group of A-automorphisms of $R$ such that

(1) $R^{G}$ is Noetherian, and

(2) there exist a $\tilde{T}^{G} R^{G}$-bimodule homomorphism $\tau: \tilde{T}^{G} R \rightarrow\left(\tilde{T}^{G} R^{\prime}\right)^{G}$ and an essential $G$-stable ideal $K$ of $R$ such that $\tau(K) \subseteq R^{G}$ and $\tau(K)$ contains a regular element of $\tilde{T}^{G} R^{G}$.

Then $R^{G}$ is affine.

Proof. Our argument is based on the proof of [17, Theorem 2], using $\tau$ instead of the $t_{\Lambda}$ used there. We first remark that as $\tilde{T}^{G} R \subseteq \tilde{T} R$, a Noetherian $R$-module by Proposition 3.2, part (2), $\tilde{T}^{G} R$ is also finite over $R$, so we may write $\tilde{T}^{G} R=\sum_{i=1}^{m} R s_{i}$, $s_{i} \in \tilde{T}^{G}$.

Also, $\tilde{T}^{G} R^{G}$ is affine over $A$. For, since $\tilde{T} R$ is finite over $\tilde{T}$ (by Proposition 3.1, part (3)), and $\tilde{T}$ is finite over $\tilde{T}^{G}$, we have $\tilde{T} R$ finite over $\tilde{T}^{G}$. As $\tilde{T}^{G}$ is Noetherian and $\tilde{T}^{G} R^{G} \subseteq \tilde{T} R$, it follows that $\tilde{T}^{G} R^{G}$ is finite over $\tilde{T}^{G}$. Thus $\tilde{T}^{G} R^{G}$ is affine, since $\tilde{T}^{G}$ is affine.

We now claim that $\tilde{T}^{G} R^{G}$ is finite over $R^{G}$. By assumption, $\tau(K)$ contains an element, say $a$, which is regular in $\tilde{T}^{G} R^{G}$. Thus $\tilde{T}^{G} R^{G} \cong \tilde{T}^{G} R^{G} a$ as $\tilde{T} R^{G}$-modules. But

$$
\tilde{T}^{G} R^{G} a \subseteq \tilde{T}^{G} R^{G} \tau(K) \subseteq \tau\left(\tilde{T}^{G} R K\right) \subseteq \tau\left(\sum_{i=1}^{m} K s_{i}\right)=\sum_{i=1}^{m} \tau(K) s_{i} .
$$

Since $\tau(K)$ is an ideal of $R^{G}$, which is assumed to be Noetherian, $\tau(K)$ is a finite $R^{G}$-module. It follows that $\tilde{T}^{G} R^{G}$ is isomorphic to a submodule of a finite $R^{G}$. module; thus $\tilde{T}^{G} R^{G}$ is finite over $R^{G}$.

In addition, we may assume that $\tilde{T}^{G} R^{G}=\sum_{i=1}^{r} R^{G} t_{i}$, where the $t_{i} \in \tilde{T}^{G}$ and so centralize $R^{G}$. Thus we may apply a version of the Artin-Tate lemma [17, Proposition 2] to conclude that $R^{G}$ is affine.

We now prove our second main theorem.

THEOREM 3.3. Let $R$ be a semiprime Noetherian PI ring, which is an affine algebra over a commutative Noetherian ring $A$. Let $G$ be a finite group of A-automorphisms of $R$ such that $R^{G}$ is Noetherian. Then $R^{G}$ is also affine over $A$, in any of the following cases:

(1) $R$ has no $|G|$-torsion, or

(2) $G$ is $X$-outer on $R$, or

(3) $R$ has no nilpotent elements.

Proof. In all three cases, it is known that $R^{G}$ is semiprime Goldie $[10,15,11$; moreover $Q\left(R^{G}\right)=Q(R)^{G}$. Thus $\tilde{T}^{G} \subseteq Q\left(R^{G}\right)$, so an element regular in $R^{G}$ is regular in $\tilde{T}^{G} R^{G}$. In addition, any essential ideal of $R^{G}$ contains a regular element. Thus it suffices to show, by Proposition 3.2, that in each case there exists suitable $\tau$ and $K$ so that $\tau(K)$ is essential in $R^{G}$. 
In cases (1) and (2), we may use $\tau=t$, the usual trace function, and $K=R$ (by Lemma 2.1). In case (3), we use the function $\tau$ constructed in Theorem 2. Clearly $\tau(R) \subseteq R^{\prime}$, and $\tau(K)$ is essential in $R^{G}$ for some essential ideal $K$ of $R$. It remains only to show that $\tau: \tilde{T}^{G} R \rightarrow\left(\tilde{T}^{G} R^{\prime}\right)^{G}$ and is a $\tilde{T}^{G} R^{G}$-module homomorphism. However, this follows from Remark 2.10, using $C_{0}=\tilde{T}^{G}$, as the extended center of $R$ is the center of $Q(R)$.

In closing, we raise the question as to whether the hypothesis in Theorem 3.3 that $R^{G}$ is Noetherian is superfluous. We know of no examples in cases (1), (2) or (3) of a semiprime, Noetherian, affine algebra $R$ and a finite group $G$ such that $R^{G}$ is not Noetherian. The examples [ 19 and 4] of non-Noetherian fixed rings are not affine; in any event, a counterexample could not be commutative.

\section{REFERENCES}

1. S. A. Amitsur, On rings of quotients, Symposia Mathematica, vol. 8, Academic Press, New York, 1972, pp. 149-164.

2. S. A. Amitsur and L. W. Small, Prime PI-rings, Bull. Amer. Math. Soc. 83 (1977), 249-251.

3. G. Bergman and I. M. Isaacs, Rings with fixed point free group actions, Proc. London Math. Soc. 27 (1973), 69-87.

4. C. L. Chuang and P. H. Lee, Noetherian rings with involution, Chinese J. Math. 5 (1977), 15-19.

5. M. Cohen, A Morita context related to finite automorphism groups of rings, Pacific J. Math. 98 (1981).

6. M. Cohen and S. Montgomery, The normal closure of a semiprime ring, Ring Theory: Proc. 1978 Antwerp Conf., Lecture Notes in Math., vol. 51, Marcel Dekker, New York, 1979, pp. 43-59.

7. _ Trace functions for finite automorphism groups of rings, Arch. Math. (Basel) 35 (1980), 516-527.

8. D. Farkas and R. Snider, Noetherian fixed rings, Pacific J. Math. 69 (1977), 347-353.

9. R. Guralnick, I. M. Isaacs and D. S. Passman, Nonexistence of partial traces for group actions, Rocky Mountain J. Math. 11 (1981), 395-405.

10. V. K. Kharchenko, Galois extensions and quotient rings, Algebra i Logika 13 (1974), 264-281.

11. __ Generalized identities with automorphisms, Algebra i Logika 14 (1975), 215-237.

12. $\ldots$ Fixed elements under a finite group acting on a semiprime ring, Algebra i Logika 14 (1975), $328-355$.

13. Galois theory of semiprime rings, Algebra i Logika 16 (1977), 313-363.

14. W. S. Martindale, Prime rings satisfying a generalized polynomial identity, J. Algebra 12 (1969), 576-584.

15. S. Montgomery, Outer automorphisms of semiprime rings, J. London Math. Soc. 18 (1978), 209-221.

16. __ A A _ _ Atomorphism groups of rings with no nilpotent elements, J. Algebra 60 (1979), 238-248.

17. S. Montgomery and L. W. Small, Fixed rings in Noetherian rings, Bull. London Math. Soc. 13 (1981), 33-38.

18. D. Mumford, Hilbert's fourteenth problem, Mathematical Developments Arising from Hilbert's Problems (edited by F. E. Browder), Proc. Sympos. Pure Math., vol. 28, Amer. Math. Soc., Providence, R. I., 1976, pp. 431-444.

19. K. R. Nagarajan, Groups acting on Noetherian rings, Nieuw Arch. Wisk. 16 (1968), 25-29.

20. W. Schelter, Affine PI algebras are catenary, J. Algebra 51 (1978), 12-18.

Department of Mathematics, Ben Gurion University of THE NegeV, BeERSheva, IsRael

Department of Mathematics, University of Southern California, los Angeles, California 90007 